\title{
Dot1l expression predicts adverse postoperative prognosis of patients with clear-cell renal cell carcinoma
}

\author{
Yang $Q u^{1, *}$, Li Liu ${ }^{1, *}$, Jiajun Wang ${ }^{1, *}$, Wei $X^{1}{ }^{1}$, Yu Xia ${ }^{1}$, Qi Bai ${ }^{1}$, Ying Xiong ${ }^{1}$, Qilai \\ Long $^{1}$, Jiejie Xu' ${ }^{2}$, Jianming Guo ${ }^{1}$ \\ ${ }^{1}$ Department of Urology, Zhongshan Hospital, Fudan University, Shanghai 200032, China \\ ${ }^{2}$ Department of Biochemistry and Molecular Biology, School of Basic Medical Sciences, Fudan University, Shanghai 200032, \\ China \\ *These authors have contributed equally to this work \\ Correspondence to: Jiejie $X u$, email: jjxufdu@fudan.edu.cn \\ Jianming Guo, email: guo.jianming@zs-hospital.sh.cn \\ Keywords: clear-cell renal cell carcinoma, Dot1l, overall survival, prognostic biomarker, nomogram
}

Received: August 15, $2016 \quad$ Accepted: September 22, $2016 \quad$ Published: October 5, 2016

\section{ABSTRACT}

Background: Disruptor of telomeric silencing 1-like (Dot1l), a histone methyltransferase that targets the histone H3 lysine 79 (H3K79), has been reported that its high expression is associated with various cancers, while the association between Dot1l expression and clear-cell renal cell carcinoma (CCRCC) is still unknown.

Patients and Methods: We retrospectively enrolled 282 patients with cCRCC undergoing nephrectomy from a single institution between 2005 and 2007, with a median follow-up of 99 months. Dot1l expression was evaluated by immunohistochemistry in clinical specimens. We compared the clinical outcomes by Kaplan-Meier survival analyses and assessed the prognostic value of Dot1l expression. Harrell's concordance index (C-index) was used to assess the predictive accuracy of different prognostic models.

Results: Higher Dot1l expression indicated poorer OS $(P<0.001)$ and RFS $(P<0.001)$ in patients with ccRCC. Moreover, Dot1l expression could stratify ccRCC patients in pT stage, Fuhrman grade and SSIGN/ Leibovich subgroups, which might redefine individual risk stratification. Multivariate analyses further indicated that Dot1l expression was an independent prognostic factor for OS $(P=0.007)$ and RFS $(P=0.001)$. The prognostic accuracy of conventional prognostic models was notably improved with Dot1l integration. Two nomograms and calibration plots were built to predict OS and RFS for patients with CCRCC and performed better based on C-index value.

Conclusion: Dot1l expression is a promising independent prognostic indicator for postoperative recurrence and survival of patients with ccRCC.

\section{INTRODUCTION}

Renal cell carcinoma (RCC), accounting for $2-3 \%$ of all human malignancies, is the most common cancer in kidney [1]. Among all histological subtypes of RCC, clear-cell renal cell carcinoma (ccRCC) is the commonest one accounting for more than $80 \%$ cases [2]. Although tremendous progress in diagnosis and treatment took place recently, radiography especially, it is still estimated that 62700 new cases and 14240 deaths will occur in the
USA in 2016 [3]. Clinical outcomes are hard to predict for ccRCC because of heterogeneity of molecular phenotype [4]. Therefore, we need new and valuable prognostic biomarkers for ccRCC, besides of classical prognostic models, such as TNM stage, Fuhrman grade, Eastern Cooperative Oncology Group performance status (ECOGPS), the Mayo clinic stage, size, grade, and necrosis score (SSIGN) [5] and the University of Los Angeles integrated staging system (UISS) [6] category systems. Furthermore, ccRCC has a high risk of metastasis (about 20-30\%) and 
patients with metastasis usually get poor outcomes, which means biomarkers are needed for treatment, either [3].

Recently, post-translational histone modification has become more and more popular in cancer research due to their ability to regulate gene transcription. By modifying chromatin structure with methyl groups, histone methylation is the first identified post-translational histone modification among the four classic histone modifications [7]. In this process, the methyl groups attach to a lysine or arginine residue first, and connect to the -amine group with the help of proper methyltransferases [8]. Among all those modifications, methylation on histone $\mathrm{H} 3$ lysine 79 (H3K79) is a crucial one and has been studied recently. Furthermore, several researches revealed that methyltransferases and demethylases played key roles in the genesis and development of ccRCC, such as EZH2 and UTX [9].

Disruptor of telomeric silencing 1-like (Dot11), located on the nucleosome surface, is the only known mammalian histone methyltransferase which targets the H3K79 position [10]. Just like its homolog gene Dot1 which first identified in yeast, Dot11 catalyzes the mono, di-, and tri- methylation of H3K79 specifically with a unique catalytic domain. According to recent findings, Dot11-mediated H3K79 methylation is associated with many biological processes including transcriptional regulation, DNA damage response, cell cycle progression, somatic reprogramming and embryonic cell development [11]. In addition, accumulating studies suggested that Dot1l plays an important role in the genesis and progression of mixed lineage leukemia (MLL) [12]. Still, several researches have shown that Dot1l takes part in the progression of many other tumors, such as lung cancer [13], colorectal cancer [14] and breast cancer [15]. As a promising biochemical target, one of the Dot11 inhibitors is already under investigation in a Phase I clinical trial in MLL patients [16].

However, the value of Dot11 in treatment and prognosis of ccRCC is still unknown as nobody studied on it. In this study, we analyzed the expression of Dot11 in ccRCC tissues by immunohistochemical analysis and dug out its association with clinicopathologic variables and clinical outcomes. Furthermore, we assessed the prognostic values of Dot1l expression and built two nomograms to predict individual risk for ccRCC patients.

\section{RESULTS}

\section{Dot1l staining intensity}

In order to find out whether the Dot11 expression is connected with tumor characters, we evaluated the Dot11 expression by immunohistochemical staining analysis in all 282 patients' samples first. As presented in Figure 1 , Dot11 was predominantly expressed in the nucleus of tumor cells. As the intensity of specific staining was different in different cases, we defined the cutoff point as 95 (H-score range: 15-261) by X-tile software used minimum $\mathrm{p}$ value method.

\section{Patient characteristics and its association with tumoral Dot1l expression}

As showed in Table 1, a total of 282 patients [196 male (69.5\%) and 86 female (30.5\%)] were enrolled in our study. The median follow-up was 99 months (range: 2.63120.47 months). The median age of patients was 55 years with a range of 15-83 years, and the median tumor size

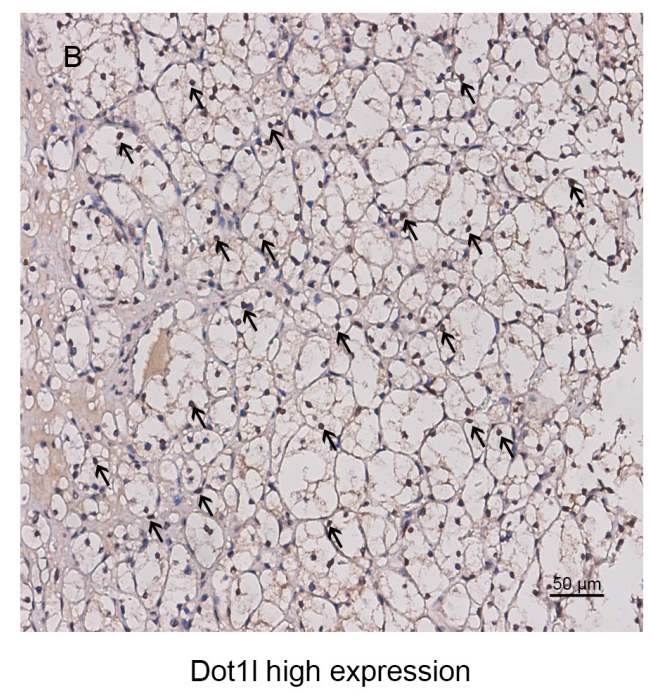

Figure 1: Dot1l expression in clear-cell renal cell carcinoma (ccRCC) tissues. Representative Dot11 immunohistochemical images with low expression level A. and high expression level B. in ccRCC tissue at $200 \times$ optical magnification. Arrows indicate positive staining of Dot11 in each image. Scale bar: $50 \mu \mathrm{m}$. 
Table 1: Clinical characteristics of patients according to tumoral dot1l expression

\begin{tabular}{|c|c|c|c|c|c|}
\hline \multirow{2}{*}{ Characteristics } & \multicolumn{2}{|c|}{ Patients } & \multicolumn{3}{|c|}{ Tumoral dot1l expression } \\
\hline & $\mathbf{n}$ & $\%$ & low & high & P-value \\
\hline All patients & 282 & 100 & 147 & 135 & \\
\hline Age, years ${ }^{\#}$ & & & & & $0.015^{*}$ \\
\hline mean $\pm \mathrm{SD}$ & & & $53.4 \pm 12.7$ & $57.1 \pm 12.8$ & \\
\hline median & & & 54 & 57 & \\
\hline range & & & $15-81$ & $27-83$ & \\
\hline Gender & & & & & $0.280 \dagger$ \\
\hline Female & 86 & 30.5 & 49 & 37 & \\
\hline Male & 196 & 69.5 & 98 & 98 & \\
\hline Tumor size, $\mathrm{cm}^{\#}$ & & & & & $0.251^{*}$ \\
\hline mean $\pm \mathrm{SD}$ & & & $4.6 \pm 2.4$ & $4.9 \pm 2.9$ & \\
\hline Median & & & 4.0 & 4.0 & \\
\hline range & & & $0.5-13.0$ & $1.0-15.0$ & \\
\hline Pathological T stage & & & & & $0.006 \%$ \\
\hline pT1 & 180 & 63.8 & 107 & 73 & \\
\hline pT2 & 28 & 10.0 & 9 & 19 & \\
\hline pT3 & 70 & 24.8 & 29 & 41 & \\
\hline pT4 & 4 & 1.4 & 2 & 2 & \\
\hline Pathological N stage & & & & & $1.000 \dagger$ \\
\hline $\mathrm{pN} 0$ & 280 & 99.3 & 146 & 134 & \\
\hline $\mathrm{pN} 1$ & 2 & 0.7 & 1 & 1 & \\
\hline Distant metastasis & & & & & $0.022 \dagger$ \\
\hline No & 267 & 94.7 & 144 & 123 & \\
\hline Yes & 15 & 5.3 & 3 & 12 & \\
\hline TNM stage & & & & & $0.001+$ \\
\hline I & 175 & 62.1 & 105 & 70 & \\
\hline II & 23 & 8.2 & 8 & 15 & \\
\hline III & 65 & 23.0 & 29 & 36 & \\
\hline IV & 19 & 6.7 & 5 & 14 & \\
\hline Fuhrman grade & & & & & $0.394+$ \\
\hline 1 & 30 & 10.6 & 17 & 13 & \\
\hline 2 & 208 & 73.8 & 109 & 99 & \\
\hline 3 & 41 & 14.5 & 20 & 21 & \\
\hline 4 & 3 & 1.1 & 1 & 2 & \\
\hline Necrosis & & & & & $0.646 \dagger$ \\
\hline Absent & 243 & 86.2 & 128 & 115 & \\
\hline Present & 39 & 13.8 & 19 & 20 & \\
\hline ECOG PS & & & & & $0.131+$ \\
\hline 0 & 206 & 73.0 & 113 & 93 & \\
\hline$\geq 1$ & 76 & 27.0 & 34 & 42 & \\
\hline UISS category & & & & & $0.006 \$$ \\
\hline low risk & 121 & 42.9 & 77 & 44 & \\
\hline mediate risk & 134 & 47.5 & 57 & 77 & \\
\hline high risk & 27 & 9.6 & 13 & 14 & \\
\hline SSIGN category & & & & & $0.001 \%$ \\
\hline $0-3$ & 217 & 77.0 & 123 & 94 & \\
\hline $4-7$ & 59 & 20.9 & 24 & 35 & \\
\hline $8+$ & 6 & 2.1 & 0 & 6 & \\
\hline
\end{tabular}

\#Split at median; *t-test for continuous variables, $\dagger \chi^{2}$ test or Fisher’s exact test, $₫$ Cochran-Mantel-Haenszel $\chi^{2}$ test, P-value $<0.05$ was regarded as statistically significant; ECOG PS, Eastern Cooperative Oncology Group performance status; UISS, UCLA Integrated Staging System; SSIGN, Mayo clinic stage, size, grade, and necrosis score. 
was $4.8 \mathrm{~cm}$ with a range of $0.5-15.0 \mathrm{~cm}$. The distribution of TNM stage at the time of surgery was I (62.1\%), II $(8.2 \%)$, III (23.0\%), and IV (6.7\%), respectively. Among all patients, 2 patients $(0.7 \%)$ were in $\mathrm{pN} 1$ stage and 15 patients $(5.3 \%)$ had been found distant metastases. Otherwise, the patients proportion of each Fuhrman grades was $1(10.6 \%), 2(73.8 \%), 3(14.5 \%)$, and $4(1.1 \%)$. In total, $13.8 \%$ cases were found necrosis at the surgery. Also, there were $27.0 \%$ patients with ECOG-PS $\geq 1$. UISS category as low risk, mediate risk, and high risk were $42.9 \%, 47.5 \%$, and $9.6 \%$ of all cases, respectively. SSIGN category classified as $0-3,4-7$ and $8+$ were $77.0 \%, 20.9 \%$ and $2.1 \%$ among all patients, respectively.

Then, we analyzed the association of those clinicopathological variables with the Dot11 expression level. With the cutoff point of 95, we defined 147 (52.1\%) patients with Dot11 low expression and 135 (47.9\%) patients with a high expression level. Patients with higher Dot1lexpression trended to be older $(P=0.015)$, have higher $\mathrm{pT} / \mathrm{pM}$ stage $(P=0.006 / 0.022)$ and have higher TNM grade $(P=0.001)$. Also, UISS and SSIGN category were found statistically significant $(P=0.006 / 0.001)$ with Dot1l expression level. The other clinicopathological characteristics had no statistically significant association with Dot11 expression level.

\section{High Dot1l expression is connected with dismal clinical outcomes of ccRCC patients}

We used Kaplan-Meier survival analysis to evaluate the clinical outcomes between different subgroups divided

A

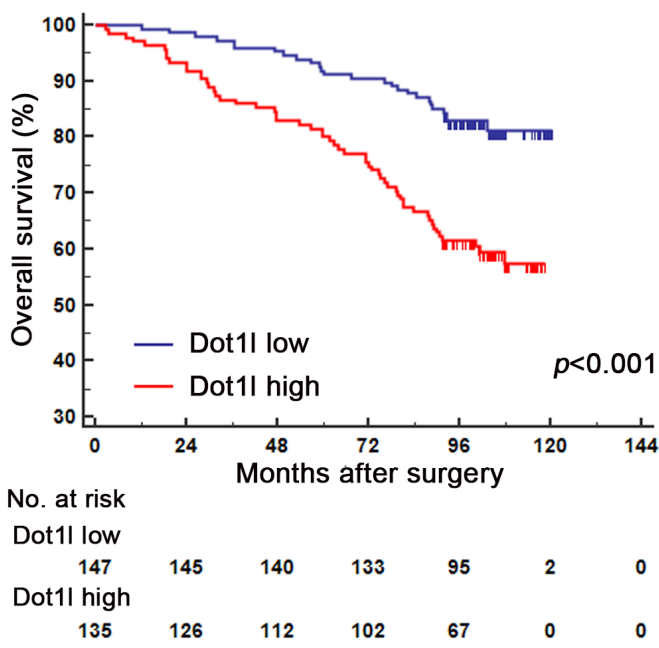

by Dot1l expression. As presented in Figure 2, patients in high Dot11 group had a worse OS $(P<0.001$, Figure 2A) and RFS $(P<0.001$, Figure $2 \mathrm{~B})$ than those in low Dot11 group. We further undertook univariate and multivariate analysis to make sure whether Dot11 is an independent predictor for ccRCC prognosis. As summarized in Table $\mathrm{S} 1$, Dot11 expression was strongly associated with clinical outcomes (OS, $P<0.001$; RFS, $P<0.001$ ) in univariate analysis. Also, pT/pM stage, Fuhrman grade, tumoral necrosis, tumor size and ECOG PS were each statistically significant in univariate analysis. We put those parameters in multivariate analysis and confirmed that Dot11 expression still was predictable for ccRCC outcomes (OS, $P=0.007$; RFS, $P=0.001)$. Together with Dot11, pT/pM stage, Fuhrman grade, tumoral necrosis and ECOG PS were all considered as independent predictors for ccRCC prognosis.

\section{Subgroup analysis for the relationship between Dot1l expression and clinical outcomes}

To make sure whether the connection of Dot11 expression with clinical outcomes of patients depends on pT-stage, Fuhrman grade and SSIGN/Leibovich score, subgroup analysis of pT-stage, Fuhrman grade and SSIGN/ Leibovich were applied respectively. As presented in Figure 3, S1 and S2, both OS and RFS strongly associated with Dot11 expression in pT $(1+2)$ group $(P<0.001$, Figure 3A, 3C), Fuhrman grade $(1+2)$ group $(P<0.001$, $P<0.001$, Figure S1A, S1C) and SSIGN/Leibovich (0-3) group $(P<0.001$, Figure S2A, S2C). In the other hand,

B

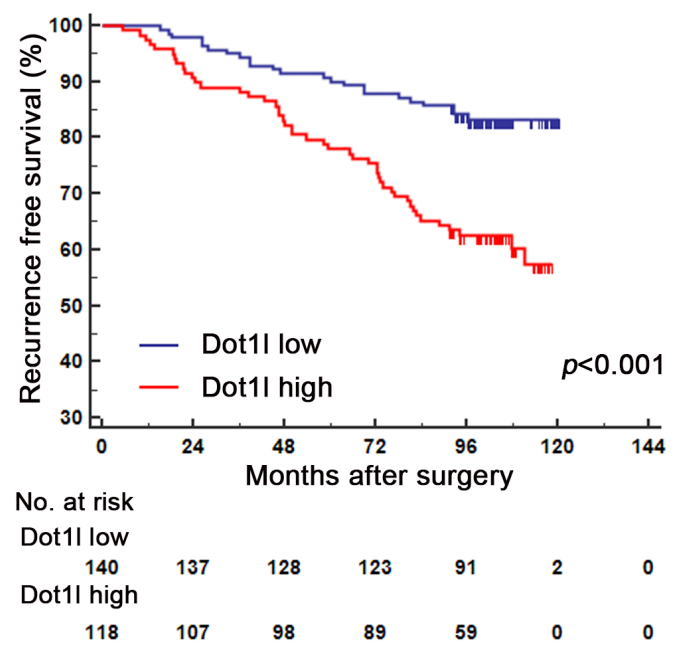

Figure 2: Kaplan-Meier analyses for overall survival (OS) and recurrence free survival (RFS) of patients with ccRCC based on Dot1l expression. Kaplan-Meier analysis of OS in ccRCC patients ( $\mathrm{n}=282)$ according to Dot11 expression A. Kaplan-Meier analysis of RFS in ccRCC patients $(\mathrm{n}=258)$ according to Dot11 expression $\mathbf{B}$. $p$-value was calculated by Log rank test, $p<0.05$ was regarded as statistically significant. 
Dot11 failed to predict tumor outcomes in $\mathrm{pT}(3+4)$ group $(P<0.001$, Figure 3B, 3D), Fuhrman grade $(3+4)$ group $(P=0.079, P=0.113$, Figure $\mathrm{S} 1 \mathrm{~B}, \mathrm{~S} 1 \mathrm{D})$ and SSIGN/ Leibovich $(\geq 4)$ group $(P<0.001$, Figure S2B, S2D).

\section{Comparison of the predictive ability between Dot1l expression and other prognostic models}

In order to further acknowledge the predictive ability of Dot11 expression, we compared Dot11 expression with

A

A $\quad$ pT stage $(1+2)$

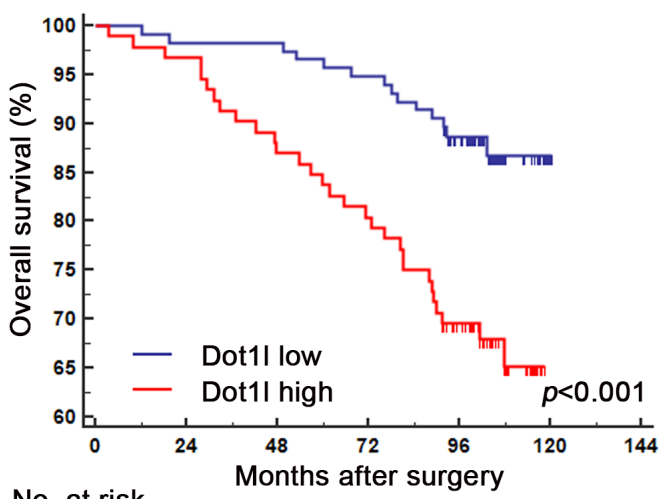

No. at risk

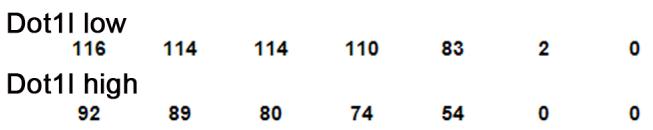

C

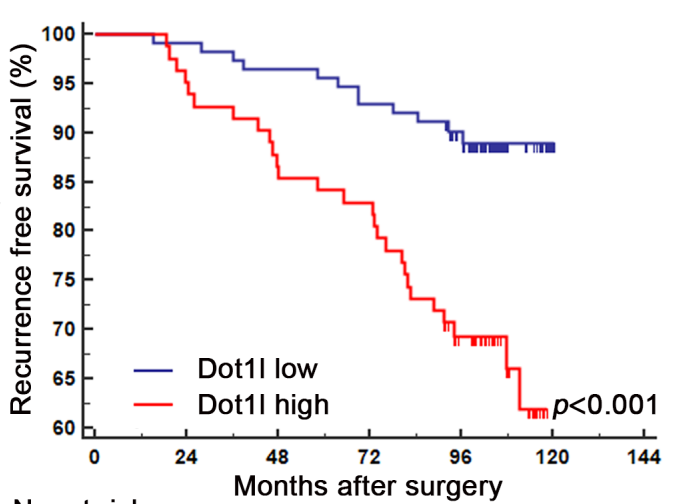

No. at risk

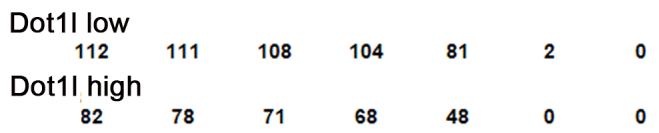

classic prognostic models, such as TNM stage, SSIGN and UISS scoring systems, respectively. Concordance index ( $C$-index) and Akaike information criteria (AIC) analysis were used for prognostic power evaluation for each model. As presented in Table 2, the $C$-indexes of TNM, SSIGN, and UISS for OS were $0.717,0.725$, and 0.727 , while Dot11 expression was 0.622. Noticeably, when Dot11 was added for OS, the $C$-index of those models was improved to $0.751,0.752$, and 0.733 respectively. More than this, the AIC value of each model combined with Dot11 was

B pT stage $(3+4)$

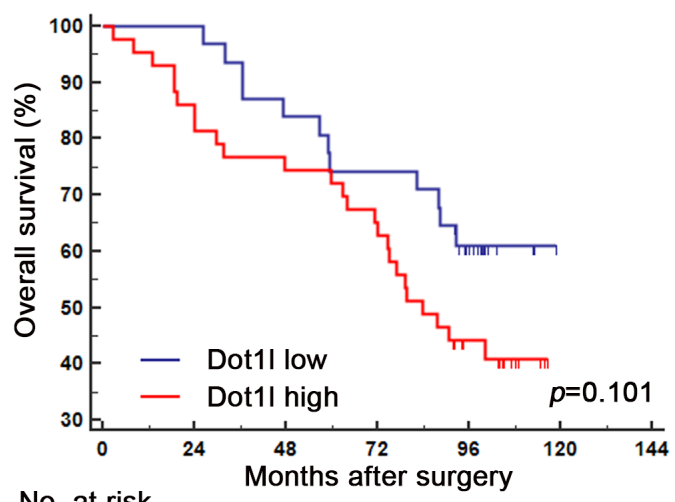

No. at risk

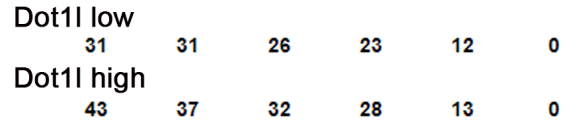

D

pT stage $(3+4)$

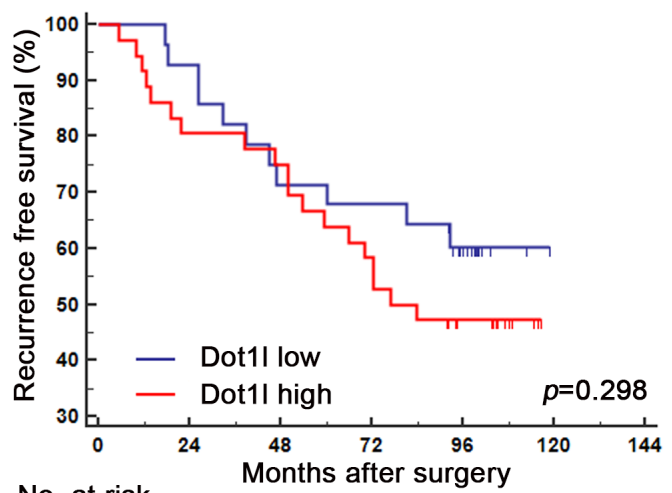

No. at risk

Dot1l low

$\begin{array}{cccccc}28 & 26 & 20 & 19 & 10 & 0 \\ \begin{array}{c}\text { Dot1l high } \\ 36\end{array} & 29 & 27 & 21 & 11 & 0\end{array}$

Figure 3: Subgroup analysis to assess prognostic value of Dot1l by pT stage in ccRCC patients. Kaplan-Meier analysis of overall survival (OS) for patients in the pT stage $(1+2)$ group A. and (3+4) group B. according to Dot11 expression; Kaplan-Meier analysis of recurrence free survival (RFS) for patients in the pT stage (1+2) group C. and (3+4) group D. according to Dot11 expression; $p$-value was calculated by Log rank test, $p<0.05$ was regarded as statistically significant. 
Table 2: Comparison of the predictive accuracy of the prognostic models

\begin{tabular}{lcccc}
\hline \multirow{2}{*}{ Models } & \multicolumn{2}{c}{ Overall survival $(\mathbf{n}=\mathbf{2 8 2})$} & \multicolumn{2}{c}{ Recurrence free survival $(\mathbf{n}=\mathbf{2 5 8})^{\mathbf{a}}$} \\
\cline { 2 - 5 } & C-index & AIC & C-index & AIC \\
\hline Tumoral Dot11 & 0.622 & 865.333 & 0.618 & 725.500 \\
TNM stage & 0.717 & 830.280 & 0.677 & 711.246 \\
TNM stage + Tumoral & 0.751 & 822.778 & 0.720 & 702.452 \\
Dot11 & 0.725 & 834.666 & 0.707 & 704.077 \\
SSIGN & 0.752 & 827.931 & 0.735 & 695.150 \\
SSIGN + Tumoral & 0.727 & 837.542 & 0.721 & 697.350 \\
Dot11 & 0.733 & 827.688 & 0.761 & 687.820 \\
UISS & 0.803 & 785.920 & 0.797 & 654.507 \\
UISS + Tumoral Dot11 & & & \\
Nomogram & &
\end{tabular}

C-index, concordance index; AIC, Akaike information criteria; SSIGN, Mayo clinic stage, size, grade, and necrosis score; UISS, UCLA Integrated Staging System. C-index were calculated from 1000 bootstrap samples to protect from overfitting.

${ }^{a}$ For recurrence-free survival analysis, 17 patients with metastasis ccRCC and 7 miss followed patients are excluded.

lower than it alone. Obviously, the $C$-index and AIC of RFS were in a similar way.

\section{Prognostic nomogram for OS and RFS in ccRCC patients}

In order to use Dot1l as a prognostic parameter, we build two nomograms for OS (Figure 4A, 4B) and RFS (Figure S3A, S3B) in ccRCC patients based on multivariate analysis. Total points were added from each point for each parameter, and could predict each patient's survival probability at different time after surgery. The calibration plots for the nomograms of OS and RFS presented good consistency between actual observation and the prediction by nomograms (Figure 4C, 4D; Figure S3C, S3D).

\section{DISCUSSION}

To our knowledge, our study is the first paper reporting the association of Dot11 expression level and clinical outcomes of ccRCC patients among more than 5600 published reports about RCC prognostic markers. By multivariate analysis, our research confirmed that Dot11 could be regarded as an independent prognostic factor for ccRCC patients. Also, we found that patient with a higher Dot1l expression tends to have a worse clinical outcome in survival analyses. In the meanwhile, Dot1l expression could stratify ccRCC patients by survival analyses in pT stage (1+2), Fuhrman grade $(1+2)$ and SSIGN (03) subgroups, which might redefine risk stratification of ccRCC patients. Furthermore, the incorporation of Dot1l expression into prognostic models such as SSIGN and UISS could dramatically enhance their prognostic accuracy. Finally, two nomograms were built to predict patients' clinical outcomes based on Dot1l and other clinicopathologic parameters. It is nice to see that our nomograms have a better predictive performance than existed prognostic models based on C-index analysis.

It is well established that epigenetic modifications play a major role in genesis and development of cancer. Among those modifications, histone methylation acts as a key step because of its contribution to cell-cycle progression, somatic reprogramming and tumor genesis [11]. Dot11, as a methyltransferase for histone H3K79 methylation, was found associations with several cancers. Barry E et al, demonstrated that Dot1l had a close connection with mixed lineage leukemia (MLL) and discussed several mechanisms [22]. In lung cancer, over expression of DOT1L leads to RNAi mediated changes which takes part in tumoral genesis [13]. In prostate cancer, Dot11 directly methylates androgen receptor to regulate its activity [23]. Besides, Zhang et al, showed that selective Dot1l inhibitors could suppress proliferation and migration in breast cancer cells [24]. Still, Dot1l has been reported to have association with other cancers, such as colorectal cancer [25]. However, the prognostic ability of Dot1l for ccRCC is still unknown. Moreover, we found that Dot1l copies had some mutations in ccRCC samples from the DNA information of 2013 TCGA cohort data, which indicated that Dot1l might be associated with ccRCC.

As the only known histone methyltransferase, which targets the histone H3K79, Dot1l adds methyl groups on histone H3K79 and generate mono- (H3K79me), di(H3K79me2) and tri-methylation (H3K79me3).Up to now, several mechanisms have been summarized to explain 
the effect of Dot11 on tumor genesis and development. Firstly, Dot11 mediated methylation of H3K79 has been implicated in transcriptional elongation and cell cycle regulation, which influence the cell division and differentiation and then generate cancer cells. Schulze $\mathrm{J}$ et al, found that the level of $\mathrm{H} 3 \mathrm{~K} 79 \mathrm{me} 2$ is different between different stages of cell cycle, while H3K79me3 remains constant throughout the cell cycle [26]. De Vos D et al, then demonstrated that H3K79me3level increases progressively in mutant cells [27]. Secondly, Dot11 has regulatory functions in gene transcription, which has a tight relationship with tumor genesis. An interesting phenomenon has been noticed that H3K79 mono- and di-methylation leads to activation of gene transcription, while H3K79 tri-methylation results in gene repression [11]. Thirdly, Dot11 has been proved to play a key role in haematopoiesis, and the high expression of Dot1l tends to cause MLL. GATA2, a growth factor essential for early haematopoiesis, was reported to be regulated by Dot11 and play a crucial role in this progress [28]. In addition, Dot11 has some other regulatory functions, which may have something to do with cancer, such as inhibition of somatic reprogramming [29] and promotion in DNA damage repair [11]. With those probable mechanisms, some Dot11 inhibitors were studied for anti-tumor treatment. Interestingly, Dot11 has a unique AdoMet binding motif for histone methylation, which is the only known non-SET domain histone methyltransferase protein [10]. This makes Dot11 to be a promising therapeutic target, and one of the Dot11 inhibitors is in phase I clinical trials [30].

However, the mechanism above cannot explain all tumors well as many downstream mechanisms are still unknown, especially for ccRCC. Our study focused on the connections between Dot1l expressions and the clinical outcomes of ccRCC patients, and confirmed the regulatory functions of Dot11 on tumor progression indirectly. According to our results, patients with early pT stage $(1+2)$ could be stratified by Dot11 expression
A

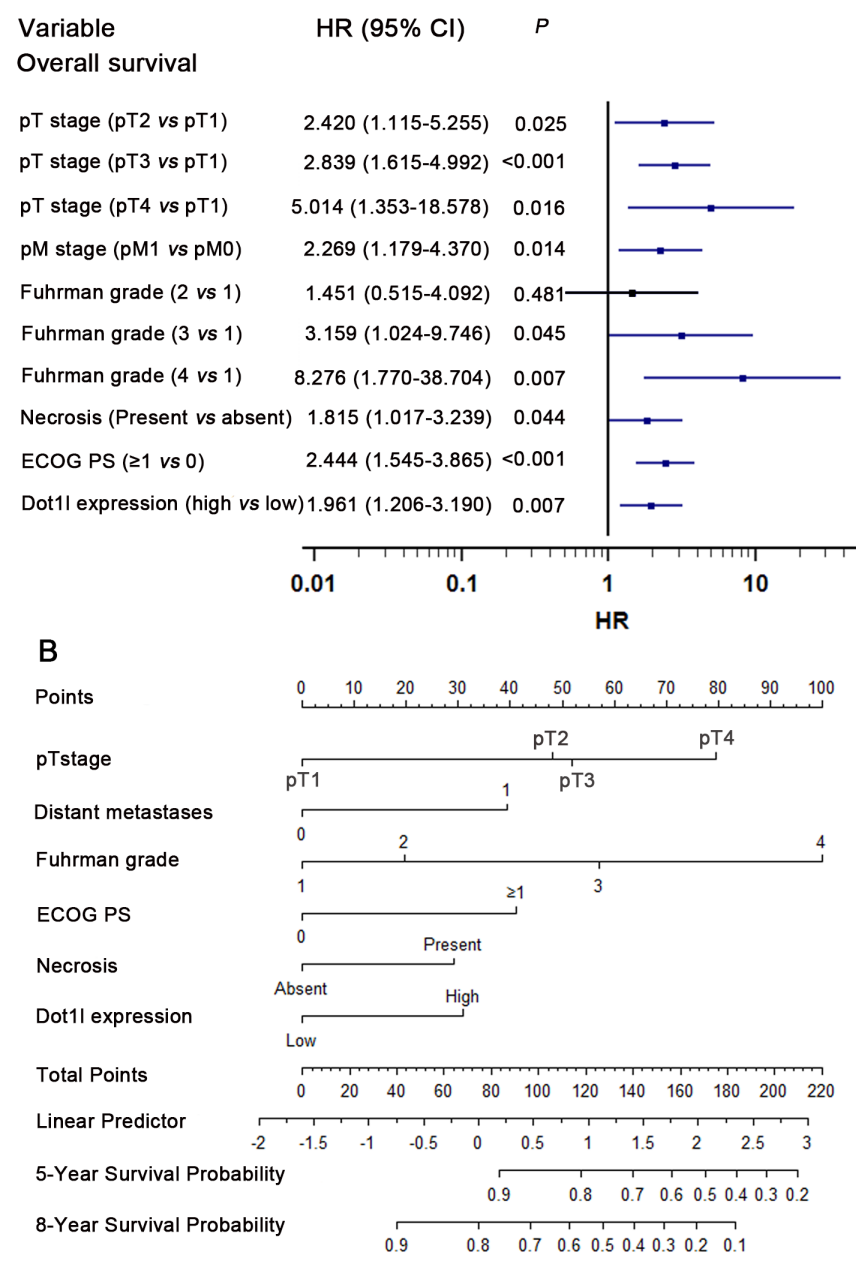

C

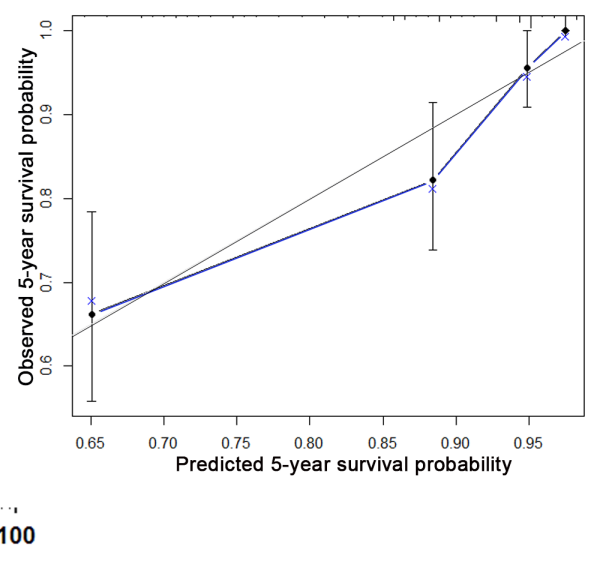

D

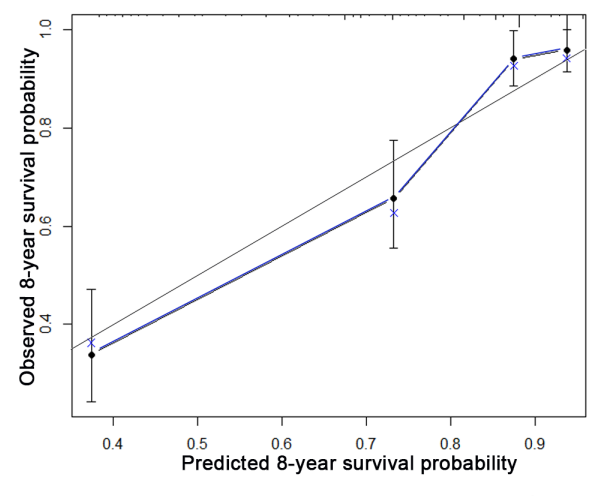

Figure 4: Multivariate analysis, Nomogram and calibration plots for prediction of overall survival (OS) in patients with ccRCC. Multivariate analysis identified independent prognostic factors of OS A. nomogram to predict OS at 5- and 8- years after nephrectomy B. the calibration plots for predicting OS at 5-years C. and 8-years D. 
while those with late pT stage $(3+4)$ could not. It is quiet similar in the SSIGN subgroup, which means that risk stratification of ccRCC patients might be redefined. Clinically, ccRCC patients with high Dot1l expression might need adjuvant therapy or a more proactive followup after surgery, even if they are low-risk patients based on classic clinicopathologic features. Furthermore, patients with better differentiation (Fuhrman grade 1+2) could be stratified by Dot11 expression, while those with higher Fuhrman grade $(3+4)$ could not, which suggested that Dot1l could be involved in the tumor differentiation.

Although our study revealed the prognostic significance of Dot11 expression ccRCC patients, some limitations remain to be acknowledged. As this is a retrospective study and all samples and data of patients were collected from a single institution, a prospective, multicenter study is needed to further validate our results. Additionally, the proportion of patients with pT4 and pN1 were insufficient in our study, which might weaken Dot1l's predictive power in those subgroups. Furthermore, the complete interaction network of Dot11 in ccRCC is still unclear and needs to be fully elucidated in future study, especially for the functional role of Dot11 in ccRCC. Finally, future studies can focus on the therapeutic effect of Dot11 on ccRCC patients, as Dot11 inhibitors have been tested for MLL treatments

\section{CONCLUSION}

In conclusion, our study indicated that Dot11 expression can be regarded as a prognostic factor for ccRCC patients. Patients with higher Dot1l expression tend to get poorer clinical outcomes. Dot1l expression could further stratify ccRCC patients in lower pT stage, Fuhrman grade and SSIGN category, which might redefine the risk stratification of ccRCC patients and guided clinical decisions. Last but not least, it is promising to explore Dot11 as a therapeutic target for ccRCC based on the crucial role of Dot11 in tumoral progression.

\section{MATERIALS AND METHODS}

\section{Patients and database}

A total of 282 patients with ccRCC who underwent nephrectomy were enrolled in base cohort retrospectively from the Department of Urology, Zhongshan Hospital, Fudan University (Shanghai, China) between Jan 2005 and Jun 2007. Clinical Research Ethics Committee of Fudan University approved the study with the approval number B2015-030 in Feb 2015 and each patient included in cohort was informed consent. The inclusion criteria for our study were conformed as follows: (a) confirmed postoperative histopathology diagnosis as ccRCC; (b) no adjuvant anticancer therapy after or before surgery and (c) no comorbidities. Patients who had histories of preoperative neoadjuvant and/or postoperative adjuvant therapy, perioperative mortalities, diagnosis of a mixture type of ccRCC and other RCC type were not included. For each patient, the following clinicopathological information was collected: age, gender, tumor size, pT stage, $\mathrm{pN}$ stage, $\mathrm{pM}$ stage, TNM stage, Fuhrman grade, presence of histological tumor necrosis, and ECOG PS. Each patient was staged with radiographic reports and postoperative pathological results, which were confirmed according to 2010 AJCC TNM classification [17]. Otherwise, ECOG PS score was calculated to each patient at the time of diagnosis. Both data were reassessed by two urologicpathologists (Yuan J. and $\mathrm{H}$. $\mathrm{Fu}$ ) independently using $\mathrm{H} \& \mathrm{E}$-stained paraffin sections. Histological subtype of RCC was defined According to the 2014 EAU guidelines [18]. Fuhrman grade was confirmed according to 2012 ISUP consensus [19].The SSIGN, UISS score were applied to evaluated patient risks according to previous papers $[5,6]$.

Overall survival (OS) was calculated from the date of nephrectomy to the time of death or the most recent follow-up, while recurrence free survival (RFS) was calculated from the time of nephrectomy to the time of recurrence, which defined as local or distant metastases confirmed by imaging, biopsy or physical examination. Patients were followed-up with physical examination, laboratory studies, chest imaging and abdominal ultrasound or CT scan every 3 months for the first 5 years and annually thereafter. The last follow-up time was Jan 30, 2015. Totally 17 patients were excluded from RFS analysis for preoperational metastases and 7 patients for missing data of recurrence state. All baseline demographic, clinical, laboratory data, radiographic and pathological reports were reconfirmed by us.

\section{Immunohistochemistry and evaluation}

Immunohistochemical staining was performed on tissue microarray (TMA) with antibody against Dot11 (ab64077, Abcam, 1:100 dilution) and proper visualization reagent (DakoEnVision Detection System) as previously described [20]. Olympus CDD camera, Nikon eclipse Ti-s microscope ( $\times 200$ magnification $)$ and NIS-Elements F3.2 software were used to record the results. The staining intensity and extent was scored by two independent pathologists without the knowledge of the patients' outcomes. A semiquantitative $\mathrm{H}$-score, ranged from 0 to 300 , was used for each sample by evaluating the staining intensities (0: negative, 1: weak, 2: moderate, 3: strong) and distribution areas (0-100\%). Three independent shots with strongest staining were selected for each core and the mean score of the three shots was regarded as the final staining intensity for each sample. The H-score cutoff point for determining tumoral Dot 11 high/low expression is 95, which was evaluated by X-tile software (Yale 
University School of Medicine, New Haven, CT, USA) using minimum $p$ value method [21].

\section{Statistical analysis}

Statistical analysis in our study was performed by SPSS 21.0 (SPSS Inc., IL, Chicago, USA), MedCalc software (version 11.4.2.0; MedCalc, Mariakerke, Belgium), Stata 12.0 (StataCorp, College Station, TX, USA) and R software version 3.1.2 with the "rms" package (R Foundation for Statistical Computing, Vienna, Austria). $\chi 2$ test, Fisher's exact method test and CochranMantel-Haenszel $\chi 2$ test were applied for categorical data, while Student's t test was used to analyze continuous variables, to assess the relationship between tumoral Dot11 expression and patients' clinical parameters. Survival (including OS and RFS) curves were made using Kaplan-Meier method and log-rank test. Univariate and multivariate Cox proportional hazard models were used to find the impact of parameters on OS and RFS. Only those statistically significant parameters showed in the univariate analysis were considered in the multivariate analysis. Harrell's concordance index (C-index) and the Akaike information criterion (AIC) value were used to assess the predictive accuracy of different prognostic models. R software with "rms" package was used to generate the nomograms and calibration plots. Parameters which were statistical significant in multivariate analyses were selected to built nomograms. All statistical tests were two sided with a statistically significant difference considered at $P<0.05$.

\section{ACKNOWLEDGMENTS}

This study was funded by grants from National Key Projects for Infectious Diseases of China (2012ZX10002012-007, 2016ZX10002018-008), National Natural Science Foundation of China (31100629, $31270863,81372755,31470794,81401988,81402082$, $81402085,81471621,81472227,81472376,31570803$, 81501999 and 81572352), Science and Technology Commission of Shanghai Municipality (14ZR 1406300), Program for New Century Excellent Talents in University (NCET-13-0146) and Scientific Projects of Shanghai (14ZR1406300). All these study sponsors have no roles in the study design, in the collection, analysis, and interpretation of data.

\section{CONFLICTS OF INTEREST}

The authors declare no conflicts of interest.

\section{Author contributions}

Y. Qu for acquisition of data, analysis and interpretation of data, statistical analysis and drafting of the manuscript; L. Liu, J. Wang, W. Xi, Y. Xia, Q. Bai, Y. Xiong and Q. Long for technical and material support; J. Xu and J. Guo for study concept and design, analysis and interpretation of data, drafting of the manuscript, obtained funding and study supervision. All authors read and approved the final manuscript.

\section{REFERENCES}

1. Escudier B, Porta C, Schmidinger M, Algaba F, Patard JJ, Khoo V, Eisen T, Horwich A. Renal cell carcinoma: ESMO Clinical Practice Guidelines for diagnosis, treatment and follow-up. Ann Oncol. 2014; 25: i49-i56.

2. Rini BI, Campbell SC, Escudier B. Renal cell carcinoma. Lancet. 2009; 373:1119-1132.

3. Siegel RL, Miller KD, Jemal A. Cancer statistics, 2016. CA Cancer J Clin. 2016; 66:7-30.

4. Jonasch E, Futreal PA, Davis IJ, Bailey ST, Kim WY, Brugarolas J, Giaccia AJ, Kurban G, Pause A, Frydman J, Zurita AJ, Rini BI, Sharma P, Atkins MB, Walker CL, Rathmell WK. State of the science: an update on renal cell carcinoma. Mol Cancer Res. 2012; 10:859-880.

5. Frank I, Blute ML, Cheville JC, Lohse CM, Weaver AL, Zincke H. An outcome prediction model for patients with clear cell renal cell carcinoma treated with radical nephrectomy based on tumor stage, size, grade and necrosis: the SSIGN score. J Urol. 2002; 168:2395-2400.

6. Leibovich BC, Blute ML, Cheville JC, Lohse CM, Frank I, Kwon ED, Weaver AL, Parker AS, Zincke H. Prediction of progression after radical nephrectomy for patients with clear cell renal cell carcinoma: a stratification tool for prospective clinical trials. Cancer-Am Cancer Soc. 2003; 97:1663-1671.

7. Allfrey VG, Faulkner R, Mirsky AE. Acetylation and methylation of histones and their possible role in the regulation of rna synthesis. Proc Natl Acad Sci U S A. 1964; 51:786-794.

8. Bannister AJ, Schneider R, Kouzarides T. Histone methylation: dynamic or static? Cell. 2002; 109:801-806.

9. Duns G, van den Berg E, van Duivenbode I, Osinga J, Hollema H, Hofstra RM, Kok K. Histone methyltransferase gene SETD2 is a novel tumor suppressor gene in clear cell renal cell carcinoma. Cancer Res. 2010; 70:4287-4291.

10. Min J, Feng Q, Li Z, Zhang Y, Xu RM. Structure of the catalytic domain of human DOT1L, a non-SET domain nucleosomal histone methyltransferase. Cell. 2003; 112:711-723.

11. Wong M, Polly P, Liu T. The histone methyltransferase DOT1L: regulatory functions and a cancer therapy target. Am J Cancer Res. 2015; 5:2823-2837.

12. McLean CM, Karemaker ID, van Leeuwen F. The emerging roles of DOT1L in leukemia and normal development. Leukemia. 2014; 28:2131-2138.

13. Kim W, Kim R, Park G, Park JW, Kim JE. Deficiency of H3K79 histone methyltransferase Dot1-like protein 
(DOT1L) inhibits cell proliferation. J Biol Chem. 2012; 287:5588-5599.

14. Kryczek I, Lin Y, Nagarsheth N, Peng D, Zhao L, Zhao E, Vatan L, Szeliga W, Dou Y, Owens S, Zgodzinski W, Majewski M, Wallner G, Fang J, Huang E, Zou W. IL-22(+) CD4(+) T cells promote colorectal cancer stemness via STAT3 transcription factor activation and induction of the methyltransferase dot11. Immunity. 2014; 40:772-784.

15. Oktyabri D, Ishimura A, Tange S, Terashima M, Suzuki T. DOT1L histone methyltransferase regulates the expression of BCAT1 and is involved in sphere formation and cell migration of breast cancer cell lines. Biochimie. 2016; 123:20-31.

16. Waters NJ, Smith SA, Olhava EJ, Duncan KW, Burton RD, O'Neill J, Rodrigue ME, Pollock RM, Moyer MP, Chesworth R. Metabolism and disposition of the DOT1L inhibitor, pinometostat (EPZ-5676), in rat, dog and human. Cancer Chemother Pharmacol. 2016; 77:43-62.

17. Barry ER, Corry GN, Rasmussen TP. Targeting DOT1L action and interactions in leukemia: the role of DOT1L in transformation and development. Expert Opin Ther Targets. 2010; 14:405-418.

18. Yang L, Lin C, Jin C, Yang JC, Tanasa B, Li W, Merkurjev D, Ohgi KA, Meng D, Zhang J, Evans CP, Rosenfeld MG. lncRNA-dependent mechanisms of androgen-receptorregulated gene activation programs. Nature. 2013; 500:598-602.

19. Zhang L, Deng L, Chen F, Yao Y, Wu B, Wei L, Mo Q, Song Y. Inhibition of histone H3K79 methylation selectively inhibits proliferation, self-renewal and metastatic potential of breast cancer. Oncotarget. 2014; 5:10665-10677. doi:10.18632/oncotarget.2496.

20. Gibbons GS, Owens SR, Fearon ER, Nikolovska-Coleska Z. Regulation of Wnt signaling target gene expression by the histone methyltransferase DOT1L. Acs Chem Biol. 2015; 10:109-114.

21. Schulze JM, Jackson J, Nakanishi S, Gardner JM, Hentrich T, Haug J, Johnston M, Jaspersen SL, Kobor MS, Shilatifard A. Linking cell cycle to histone modifications: SBF and H2B monoubiquitination machinery and cellcycle regulation of H3K79 dimethylation. Mol Cell. 2009; 35:626-641.
22. De Vos D, Frederiks F, Terweij M, van Welsem T, Verzijlbergen KF, Iachina E, de Graaf EL, Altelaar AF, Oudgenoeg G, Heck AJ, Krijgsveld J, Bakker BM, van Leeuwen F. Progressive methylation of ageing histones by Dot1 functions as a timer. Embo Rep. 2011; 12:956-962.

23. Vlaming $H$, van Leeuwen $F$. The upstreams and downstreams of H3K79 methylation by DOT1L. CHROMOSOMA. 2016.

24. Onder TT, Kara N, Cherry A, Sinha AU, Zhu N, Bernt KM, Cahan P, Marcarci BO, Unternaehrer J, Gupta PB, Lander ES, Armstrong SA, Daley GQ. Chromatin-modifying enzymes as modulators of reprogramming. Nature. 2012; 483:598-602.

25. Waters NJ, Smith SA, Olhava EJ, Duncan KW, Burton RD, O'Neill J, Rodrigue ME, Pollock RM, Moyer MP, Chesworth R. Metabolism and disposition of the DOT1L inhibitor, pinometostat (EPZ-5676), in rat, dog and human. Cancer Chemother Pharmacol. 2016; 77:43-62.

26. Kim SP, Alt AL, Weight CJ, Costello BA, Cheville JC, Lohse C, Allmer C, Leibovich BC. Independent validation of the 2010 American Joint Committee on Cancer TNM classification for renal cell carcinoma: results from a large, single institution cohort. J Urol. 2011; 185:2035-2039.

27. Ljungberg $\mathrm{B}$, Bensalah $\mathrm{K}$, Canfield $\mathrm{S}$, Dabestani $\mathrm{S}$, Hofmann F, Hora M, Kuczyk MA, Lam T, Marconi L, Merseburger AS, Mulders P, Powles T, Staehler M, Volpe A, Bex A. EAU guidelines on renal cell carcinoma: 2014 update. Eur Urol. 2015; 67:913-924.

28. Delahunt B, Cheville JC, Martignoni G, Humphrey PA, Magi-Galluzzi C, McKenney J, Egevad L, Algaba F, Moch H, Grignon DJ, Montironi R, Srigley JR. The International Society of Urological Pathology (ISUP) grading system for renal cell carcinoma and other prognostic parameters. Am J Surg Pathol. 2013; 37:1490-1504.

29. Bai Q, Liu L, Xia Y, Long Q, Wang J, Xu J, Guo J. Prognostic significance of ST3GAL-1 expression in patients with clear cell renal cell carcinoma. Bmc Cancer. 2015; 15:880.

30. Camp RL, Dolled-Filhart M, Rimm DL. X-tile: a new bioinformatics tool for biomarker assessment and outcomebased cut-point optimization. Clin Cancer Res. 2004; 10:7252-7259. 